\title{
Difficulties encountered managing nodules detected during a computed tomography lung cancer screening program
}

\author{
Giulia Veronesi, MD, ${ }^{a}$ Massimo Bellomi, MD, ${ }^{\mathrm{b}, \mathrm{c}}$ Paolo Scanagatta, MD, ${ }^{\mathrm{a}}$ Lorenzo Preda, MD, ${ }^{\mathrm{b}}$ Cristiano Rampinelli, MD, ${ }^{\mathrm{b}}$ \\ Juliana Guarize, MD, ${ }^{a}$ Giuseppe Pelosi, PhD, ${ }^{c, d}$ Patrick Maisonneuve, $\mathrm{ScD},{ }^{\mathrm{e}}$ Francesco Leo, $\mathrm{MD},{ }^{a}$ Piergiorgio Solli, MD, \\ Michele Masullo, MD, ${ }^{d}$ and Lorenzo Spaggiari, $\mathrm{PhD}^{\mathrm{a}, \mathrm{c}}$
}

Earn CME credits at http:// cme.ctsnetjournals.org
From the Departments of Thoracic Surgery and Radiology, ${ }^{\mathrm{b}}$ European Institute of Oncology, Milan, Italy; School of Medicine, University of Milan, ${ }^{\mathrm{c}}$ Milan, Italy; and Department of Pathology ${ }^{\mathrm{d}}$ and Department of Epidemiology and Biostatistics, European Institute of Oncology, Milan, Italy.

Presented at the $33^{\text {rd }}$ Western Thoracic Surgical Association Meeting, June 27-30, 2007, Santa Ana Puablo, New Mexico (P. Scanagatta, MD).

Received for publication June 29, 2007; revisions received Jan 20, 2008; accepted for publication Feb 7, 2008.

Address for reprints: Giulia Veronesi, MD, Division of Thoracic Surgery, European Institute of Oncology, Via Ripamonti 435, I-20141, Milan, Italy (E-mail: giulia. veronesi@ieo.it).

J Thorac Cardiovasc Surg 2008;136:611-7 $0022-5223 / \$ 34.00$

Copyright $\odot 2008$ by The American Association for Thoracic Surgery

doi:10.1016/j.jtcvs.2008.02.082
Objective: The main challenge of screening a healthy population with low-dose computed tomography is to balance the excessive use of diagnostic procedures with the risk of delayed cancer detection. We evaluated the pitfalls, difficulties, and sources of mistakes in the management of lung nodules detected in volunteers in the Cosmos single-center screening trial.

Methods: A total of 5201 asymptomatic high-risk volunteers underwent screening with multidetector low-dose computed tomography. Nodules detected at baseline or new nodules at annual screening received repeat low-dose computed tomography at 1 year if less than $5 \mathrm{~mm}$, repeat low-dose computed tomography 3 to 6 months later if between 5 and $8 \mathrm{~mm}$, and fluorodeoxyglucose positron emission tomography if more than $8 \mathrm{~mm}$. Growing nodules at the annual screening received low-dose computed tomography at 6 months and computed tomography-positron emission tomography or surgical biopsy according to doubling time, type, and size.

Results: During the first year of screening, 106 patients underwent lung biopsy and 91 lung cancers were identified $(70 \%$ were stage I). Diagnosis was delayed (falsenegative) in 6 patients (stage IIB in 1 patient, stage IIIA in 3 patients, and stage IV in 2 patients), including 2 small cell cancers and 1 central lesion. Surgical biopsy revealed benign disease (false-positives) in 15 cases (14\%). Positron emission tomography sensitivity was $88 \%$ for prevalent cancers and $70 \%$ for cancers diagnosed after first annual screening. No needle biopsy procedures were performed in this cohort of patients.

Conclusion: Low-dose computed tomography screening is effective for the early detection of lung cancers, but nodule management remains a challenge. Computed tomography-positron emission tomography is useful at baseline, but its sensitivity decreases significantly the subsequent year. Multidisciplinary management and experience are crucial for minimizing misdiagnoses.

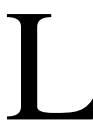

ung cancer is the most common cause of cancer death in developed countries. ${ }^{1}$ Low survival for the disease is mainly due to advanced stage of disease at diagnosis. $^{2}$ Screening with low-dose computed tomography (LD-CT) in highrisk subjects detects stage 1 disease, when it is curable by surgery, in a high proportion of cases, with $88 \%$ reported 10 -year survival. ${ }^{3}$ LD-CT screening thus seems to be a promising method for reducing lung cancer mortality. However, the technique also identifies large numbers of indeterminate nodules, many of which are inflammatory or otherwise benign, ${ }^{4}$ yet small malignant lesions can be misdiagnosed. ${ }^{5}$ The management of patients with such nodules is a challenge for the clinicians concerned with screening and nodule evaluation, and few studies have addressed the difficulties involved. ${ }^{6,7}$

LD-CT screening should diagnose a high proportion of stage 1 disease amenable to curative surgery with low morbidity and mortality, with low rates of overtreatment for 


\section{Abbreviations and Acronyms}

$\mathrm{CAD}=$ computer-aided detection

CT-PET $=$ computed tomography-positron emission tomography

LD-CT = low-dose computed tomography

benign nodules to thereby optimize cost-effectiveness. ${ }^{8}$ To achieve this, the initially adopted screening protocol must be continuously examined and improved in the light of experience gained in the management of patients with nodules. The aim of this study was to analyze the diagnostic difficulties encountered during the first 2 years of our ongoing 5-year Cosmos ${ }^{9}$ screening study for lung cancer using LD-CT and to explore the utility of computer-aided detection (CAD) volumetry and positron emission tomography (PET) to improve the diagnostic accuracy of the protocol.

\section{Materials and Methods}

Between October of 2004 and October of 2005, 5200 asymptomatic high-risk (smoking history of $\geq 20$ pack-years) individuals aged 50 years or more were enrolled in our 5-year single-center trial (Cosmos) and underwent baseline multidetector LD-CT screening for lung cancer. The subsequent year, 4815 individuals (93\%) presented for the first annual LD-CT. The screening protocol, enrollment criteria, LD-CT settings, and diagnostic algorithm have been described. ${ }^{9,10}$ The study was approved by the ethics committee of our institute, and patients were enrolled after signing the apposite consensus.

Briefly, patients with noncalcified nodules detected at baseline or new nodules $5 \mathrm{~mm}$ or less detected at annual screening were scheduled for repeat CT 1 year later. Patients with nodules between 5.1 and $8 \mathrm{~mm}$ were scheduled for repeat CT 3 to 6 months later. Patients with nodules greater than $8.1 \mathrm{~mm}$, or growing lesions less than $8 \mathrm{~mm}$ after repeat scan, were scheduled for CT-PET. Lesions suspicious for malignancy (growing or CT-PET positive) were scheduled for surgical biopsy and additional interventions. Further investigations (repeat LD-CT 6 months later, CT-PET, or surgical biopsy) for patients with growing nodules at subsequent annual screening depended on type (non-solid, solid, or partially solid), doubling time, and size of nodules. In Table 1 we report the evolution of diagnostic algorithm in relation to the time of observation for indeterminate nodules detected at baseline.

To assess the effectiveness of this protocol, we defined false-positives as benign nodules undergoing surgical biopsy and false-negatives as prevalent nodules diagnosed as lung cancer stage greater than 1 at the second annual screening. CT-PET sensitivity and specificity for baseline cancers or cancers detected after annual screening were also compared.

LD-CT scans of patients with delayed diagnosis because of protocol failure (false-negatives) were retrospectively assessed using CAD and volumetry system ${ }^{11}$ (available since December 1, 2006, at the European Institute of Oncology [EIO], Milan). Doubling times were determined automatically and compared with manual calculations based on the maximum diameter of the nodules (measured with an electronic caliper).

The CT equipment was a High Speed Advantage (General Electric, Milwaukee, Wis) with multidetector (8 or 16-slice) LD-CT scans obtained with settings at $140 \mathrm{kVp}, 30 \mathrm{~mA}, 1.75: 1$ pitch ratio, and $2.5-\mathrm{mm}$ slice thickness. Native digital imaging and communication in medicine images were processed using the lung V-Care CAD system (General Electric). The system automatically detects and segments potential regions of interest, flagging them on the CT image. Nodules not detected automatically were flagged manually. Nodules were extracted automatically using a 3-dimensional template method, and characteristics (volume and shape) were compared in sequential scans.

\section{Statistical Methods}

The utility of CT-PET was assessed by determining the sensitivity, specificity, and accuracy. Fisher's exact test was used to compare the sensitivity, specificity, and accuracy of CT-PET for baseline nodules and prevalent/incident nodules undergoing CT-PET after the first annual screening scan. We compared the manually and automatically calculated values of the nodules' doubling time by means of a scatter plot and correlation coefficient. Doubling times for stage I versus stage II to IV cancers were compared using nonparametric test for median. Survival was represented by the Kaplan-Meier method: $95 \%$ confidence intervals were calculated.

\section{Results}

To February of 2007, when almost all compliant volunteers had completed the baseline and first annual screening, the rate of patients with at least 1 noncalcified nodule was $43 \%$

TABLE 1. Clinical characteristics of 7 patients with delayed lung cancer diagnosis

\begin{tabular}{|c|c|c|c|c|c|c|c|c|c|c|}
\hline Patient & $\begin{array}{c}\text { Size baseline } \\
(\mathrm{mm})\end{array}$ & $\begin{array}{c}\text { Size } 3 \\
\text { mo (mm) }\end{array}$ & $\begin{array}{c}\text { Size } 12 \\
\text { mo (mm) }\end{array}$ & $\begin{array}{l}\text { Doubling } \\
\text { time (d) }\end{array}$ & SUV 1 y & SUV 2 y & Treatment & Tumor type & Stage & Status \\
\hline $1^{\mathrm{a}}$ & 20.9 & 20.9 & 26.8 & 341 & 1.6 & $2.2-4.42$ & CT & ADC & IV & AWD \\
\hline 2 & 4.0 & Not done & 16.0 & NA & NA & NA & CT & SCLC & IV & AWD \\
\hline 3 & 3.7 & Not done & 13.3 & 65.4 & NA & 7.51 & CT & SCLC & IV & DOD \\
\hline 4 & 7.4 & 7.4 & 10 & 284.7 & NA & 6.21 & CT-S & ADC & IIIA & AWD \\
\hline 5 & 8 & 8 & 10 & 372.8 & NA & 5.42 & $\mathrm{~S}-\mathrm{CT}$ & $A D C$ & IIB & NED \\
\hline 6 & 5.5 & 6.6 & 20 & 66.9 & 1.7 & 3.18 & CT-RT & SCC & IIIA & NED \\
\hline 7 & 4.2 & Not done & 10.9 & 89.2 & NA & 3.71 & CT-S & SCC & IIIA & NED \\
\hline
\end{tabular}

$A W D$, Alive with disease; $N E D$, not evidence of disease; $D O D$, death of disease; $C T$, chemotherapy; $R T$, radiotherapy; $S$, surgery; $A D C$, adenocarcinoma; $S C C$, squamous cell carcinoma; $N A$, not available. ${ }^{a}$ This patient refused further work-up and treatment after detection of this prevalent nodule. 


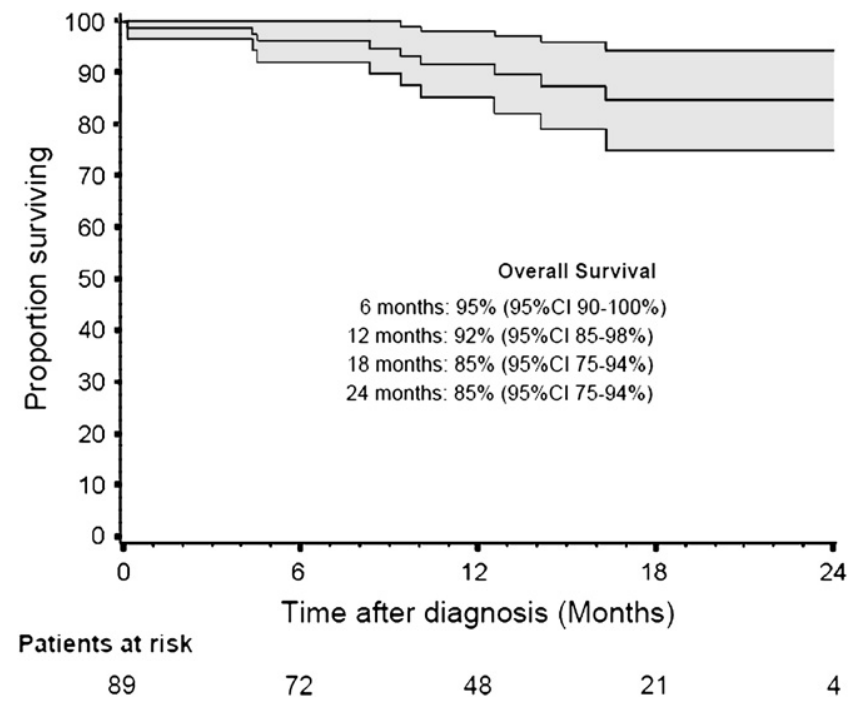

Figure 1. Overall survival in 89 screening-detected lung cancers. Cl, Confidence interval.

at baseline. A total of 106 invasive diagnostic procedures had been performed. Fifteen were benign lesions (14.4\%), and 91 were lung cancers; 64 (70\%) of these cases were stage I disease. Radical surgery was achieved in 79 patients $(89 \%)$, and 24-month survival was $85 \%$ (95\% confidence interval, $75-$ 94) (Figure 1).

\section{Delayed Diagnosis}

In 7 patients with a baseline nodule, lung cancer progressed beyond stage I during the subsequent year (false-negative), in 6 cases because of diagnostic limit of the protocol (falsenegative cases) and in 1 case because the patient refused treatment (Table 1). Figure 2 shows false-negative CT images obtained during the baseline and first annual screening. Three patients had a baseline nodule $5 \mathrm{~mm}$ or less that had progressed at the subsequent scan (scheduled, according to the protocol, for 1 year later). In 2 cases, progression was to stage IV (both small cell lung cancers; 1 patient is dead of disease and the other is alive with disease under chemotherapy); in 1 case, the progression was to stage IIIA (non-small cell, with 1 mediastinal node involved; radical multimodality treatment was applied).

Baseline lesions between 5 and $8 \mathrm{~mm}$ progressed during the subsequent year in 3 patients. Two patients underwent LD-CT 3 months after baseline, but the lesions were judged stable and scheduled, according to the protocol, to 9 months follow up, when the scheduled first annual screening scan was performed. At this annual screening scan, a secondary pleural lesion was evident in 1 case (T4N0M0, treated with surgical biopsy and definitive chemotherapy), whereas 1 other case had peribronchial lymph node metastasis (stage T2N1M0 treated with surgery and adjuvant chemotherapy). The third patient, with a suspicious central lesion, underwent repeat LD-CT at 3 and 6 months, and then CT-PET with a negative result (standard uptake value 1.7). After a further 6 months (annual screening scan), the disease had progressed to stage IIIA with a single mediastinal node station involved; the patient was treated with chemotherapy and radiotherapy. Among those with a baseline nodule greater than $8 \mathrm{~mm}$, only 1 progressed beyond stage $\mathrm{I}$ in the subsequent year: The nodule was a PET-negative ground-glass opacity. The patient refused the proposed treatment, and contralateral

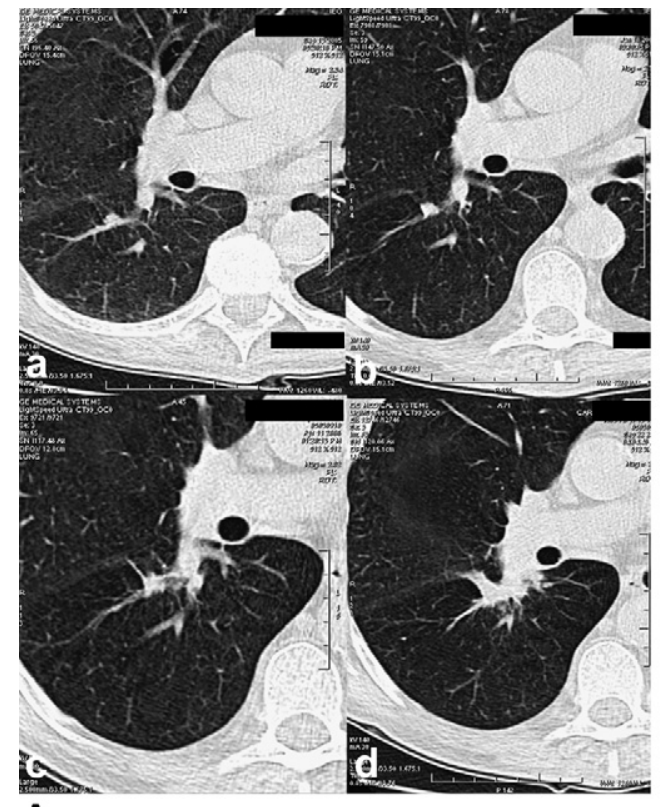

A

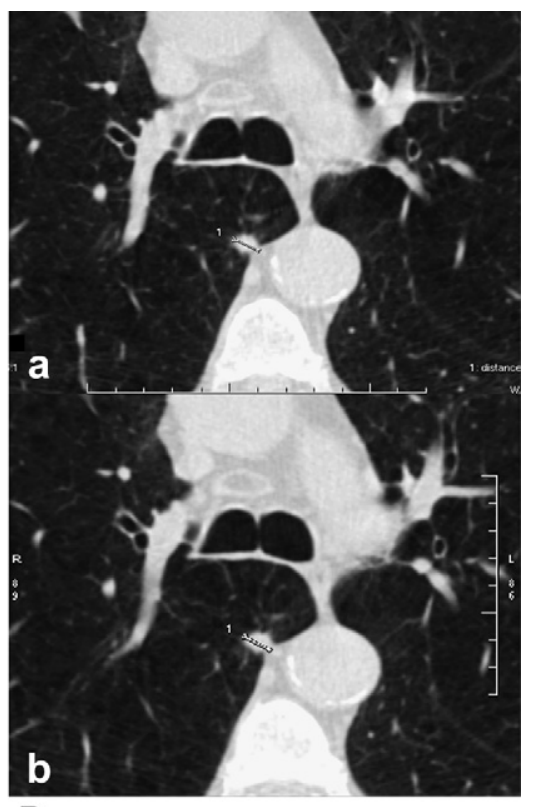

B
Figure 2. Two false-negatives arising from the diagnostic protocol. A-D, LDCT images of a centrally located lesion of the right lower lobe taken every 3 months. CT-PET after the second LD-CT was negative (SUV < 2). The lesion was diagnosed as cancer from the fourth LD-CT scan. A and B, A 7-mm lesion of the right upper lobe that remained stable in maximum diameter at 3 months follow-up LD-CT after baseline scan. Nine months after follow-up, the screening scan shows a small increase in diameter, indicating cancer. At surgery, the lesion was T2N1 squamous cell carcinoma. 


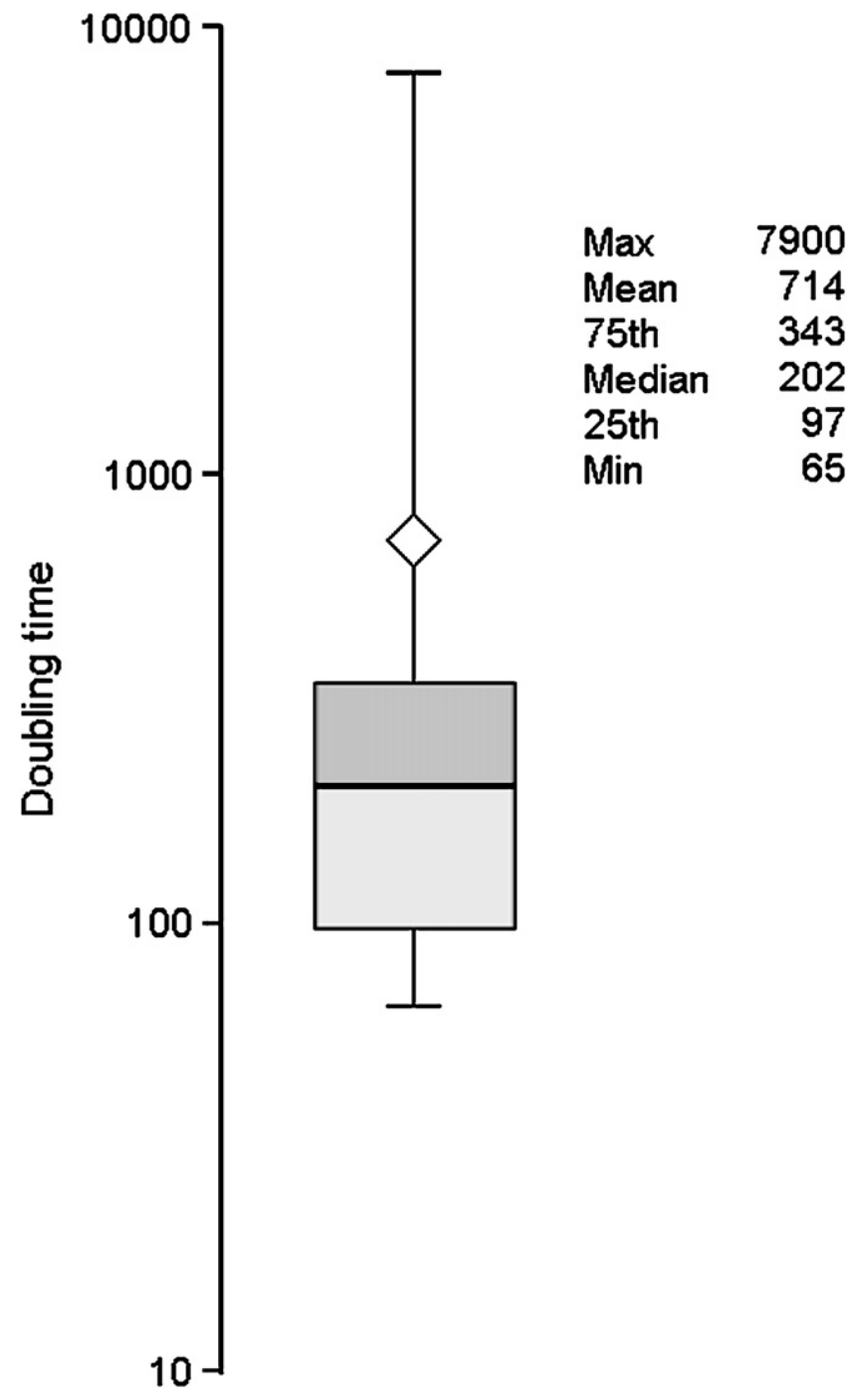

Figure 3. Distribution of the volume doubling time of 24 lung cancers diagnosed as malignant after first annual screening but detected at baseline. The extreme value of 7900 days represents a patient with a tumor stable in size that showed increased density.

lung cancer and 1 location of distant skin metastasis developed in the subsequent year.

\section{Computer-aided Detection and Volumetry}

We retrospectively assessed nodules using CAD and volumetry in 24 patients with a baseline nodule diagnosed as malignant at the next screening scan. Median manually calculated doubling time was 202 days (range 65-7900 days), as represented in Figure 3. Doubling time could be calculated automatically with the CAD system in only 13 cases. Comparison of manually and automatically calculated doubling times showed a poor correlation $\left(\mathrm{R}^{2}=0.0018\right)$.
In 1 of the 3 patients with a baseline nodule between 5 and $8 \mathrm{~mm}$ and progression beyond stage I, CAD demonstrated an increase in volume between scans 3 months apart, in the year before diagnosis (increase 559-662 $\mathrm{mm}^{3}$, doubling time 369 days). In the other 2 cases, automatic nodule segmentation was impossible because of pleural adherence in 1 case and central position adherent to bronchial and vascular structures in 1 case.

Doubling times, calculated at 1 year, of the 6 prevalent nodules that progressed beyond stage I were not significantly greater than those of prevalent nodules diagnosed as stage I at the next screening scan (187 vs 202 days; $P=.90$ ).

\section{Sensitivity and Specificity of Computed Tomography- Positron Emission Tomography}

Seventeen of the 91 CT-PET scans performed in patients with lung malignancies were false-negative. Overall CT-PET sensitivity was $81 \%$. Specificity and positive and negative predictive values were $93 \%, 89 \%$, and $94 \%$, respectively; there were 15 false-positive nodules (Table 2). Sensitivity was $88 \%$ for prevalent nodules and $70 \%$ for cancers detected after annual screening (Table 3). For cancers detected at the annual screening, CT-PET sensitivity tends to be lower ( $80 \%$ vs $65 \%)$ in prevalent nodules (nodules present at baseline) than in cancers detected "de novo" (nodules not present at baseline) (Table 4). For CT-PET-negative cancers, the median nodule size was $8.7 \mathrm{~mm}$ compared with $13 \mathrm{~mm}$ for CT-PET-positive cancers. The median doubling time was 233 days in PET-negative cancers and 116 days in PET-positive cancers.

\section{False-Positives}

Benign lesions were diagnosed at surgery (false-positives) in 15 patients (14\% of surgical cases). Pathologic findings were chronic inflammation in 7 cases, hamartoma in 3 cases, lymphoid hyperplasia in 3 cases, and other findings in 2 cases. CT-PET was positive in 8 of these cases (false-positives). Surgical treatment was preferred in all growing lesions, even in PET-negative cases. Table 2 shows the characteristics of false-positive nodules. Surgery for benign disease occurred more often at annual screening nodules (19\% of surgically treated cases) than baseline nodules (11\% of surgically treated cases). These nodules were frequently PET negative and smaller in size than baseline nodules, and more often had a histologic diagnosis of chronic inflammation. Figure 4 shows some growing false-positive lesions subject to surgical biopsy.

\section{Discussion}

The main obstacles to the large-scale dissemination of LDCT screening for persons at high risk of developing lung cancer are overdiagnosis ${ }^{4,12}$ and overuse of invasive procedures, with increased screening costs and risks of morbidity for those with benign disease. In addition, reduction of mortality in a screened population has not been demonstrated, ${ }^{13}$ mainly 
TABLE 2. Characteristics of false-positive lung nodules at years 1 and 2 of computed tomography screening and type of surgery

\begin{tabular}{|c|c|c|c|}
\hline & $\begin{array}{l}\text { Overall after baseline and } \\
\text { annual screening }\end{array}$ & After baseline screening & After annual screening \\
\hline No. in relation to total surgical cases & $15 / 104$ & $7 / 62$ & $8 / 42$ \\
\hline No. in relation to total screened & $15 / 5200$ & $7 / 5200$ & $8 / 4816(0.17 \%)$ \\
\hline subjects & $(0.29 \%)$ & $(0.13 \%)$ & \\
\hline CT-PET positive & $8 / 15$ & $5 / 7$ & $3 / 8$ \\
\hline Growing & $7 / 15$ & $1 / 7^{\mathrm{a}}$ & $6 / 8$ \\
\hline Mean size (mm) & 16.5 & 20.3 (range 8-34) & 13.1 (range 8.6-25) \\
\hline \multicolumn{4}{|l|}{ Diagnoses } \\
\hline Hamartoma & $3 / 15$ & $2 / 7$ & $1 / 8$ \\
\hline Chronic inflammation & $7 / 15$ & $2 / 7$ & $5 / 8$ \\
\hline Lymphoid hyperplasia & $3 / 15$ & $2 / 7$ & $1 / 8$ \\
\hline Other & $2 / 15$ & $1 / 7$ & $1 / 8$ \\
\hline Wedge in VATS & $5 / 15$ & $1 / 7$ & $4 / 8$ \\
\hline Wedge in thoracotomy & $9 / 15$ & $6 / 6$ & $3 / 8$ \\
\hline Lobectomy & $1 / 15$ & $0 / 7$ & $1 / 8$ \\
\hline
\end{tabular}

VATS, Videothoracoscopic approach; CT-PET, computed tomography-positron emission tomography. ${ }^{\text {a }}$ Growing at 3 months.

because of the long follow-up required by the ongoing randomized trial. $^{14}$

Although overdiagnosis may be a feature of all screening programs, clinical, ${ }^{15-17}$ biological, $^{18,19}$ and molecular data $^{20,21}$ suggest that it is less important in lung cancer than other cancers, although not all authors agree. ${ }^{13}$

In regard to the fear of invasiveness of the screening process among patients with benign disease, we conceived the present trial to use noninvasive procedures until reasonable suspicion of cancer was obtained. Basically, this meant using CT-PET for suspicious nodules $(>8 \mathrm{~mm}$, or growing lesions $\leq 8 \mathrm{~mm}$ after repeat scan), whereas many other trials resort to fine-needle aspiration biopsy. ${ }^{6}$ We recently reported an assessment (American Society of Clinical Oncology 2007) of the results obtained during the first year (after baseline screening) of the study. We

TABLE 3. Sensitivity, specificity, and accuracy of computed tomography-positron emission tomography: Overall results compared with nodules detected at baseline screening and computed tomography-positron emission tomography outcome for nodules detected at annual screening

\begin{tabular}{lcccc}
\hline & $\begin{array}{c}\text { Overall } \\
\text { baseline } \\
\text { and annual } \\
\text { screening }\end{array}$ & $\begin{array}{c}\text { During } \\
\text { baseline } \\
\text { screening }\end{array}$ & $\begin{array}{c}\text { During } \\
\text { annual } \\
\text { screening }\end{array}$ & $\begin{array}{c}\boldsymbol{P} \text { value } \\
\text { baseline } \\
\text { vs annual }\end{array}$ \\
\hline Sensitivity & $81 \%$ & $88 \%$ & $70 \%$ & .049 \\
Specificity & $93 \%$ & $94 \%$ & $87 \%$ & .37 \\
Accuracy & $88 \%$ & $92 \%$ & $77 \%$ & .007 \\
\hline
\end{tabular}

Sensitivity and accuracy of CT-PET was significantly reduced for cancers diagnosed at first annual screening compared with those diagnosed at baseline screening. found that CT-PET had good sensitivity (88\%) and specificity (94\%) for these prevalent lesions, and only $14 \%$ of screened subjects who underwent invasive diagnostic procedures had benign disease. ${ }^{9}$

The aim of the present study was to analyze in detail the reasons for protocol failures in the first year of our singlecenter lung cancer screening study. The present study shows a small percentage of delayed cancer diagnoses. Most (50\%) delayed cancers arose from 5- to 8-mm nodules. Our protocol scheduled these nodules to repeat LD-CT at 3 months. However, visual assessment of growth during this short period of time failed to identify very small differences in size. Retrospective evaluation with CAD in these cases found doubling times suspicious for cancer in approximately one third of 5to $8-\mathrm{mm}$ nodules that progressed beyond stage I.

In addition, some nodules, particularly the non-solid type, may present a stable size but increased density over time. For this reason, we have introduced the variation of the density in the diagnostic algorithm as a potential indication to surgical biopsy.

TABLE 4. Computed tomography-positron emission tomography outcomes for cancers diagnosed after annual screening

\begin{tabular}{lccc}
\hline & $\begin{array}{c}\text { Annual screening } \\
\text { incident nodules }\end{array}$ & $\begin{array}{c}\text { Annual screening } \\
\text { prevalent nodules }\end{array}$ & $\begin{array}{c}\boldsymbol{P} \text { value incident } \\
\text { vs prevalent }\end{array}$ \\
\hline Sensitivity & $80 \%$ & $65 \%$ & .68 \\
Specificity & $88 \%$ & $87 \%$ & 1.00 \\
Accuracy & $83 \%$ & $74 \%$ & .78 \\
\hline
\end{tabular}

CT-PET sensitivity tends to be lower ( $80 \%$ vs $65 \%$ ) in prevalent cancers (nodules present at baseline but diagnosed as cancer 1 year later) than in cancers detected "de novo" (nodules not present at baseline). 


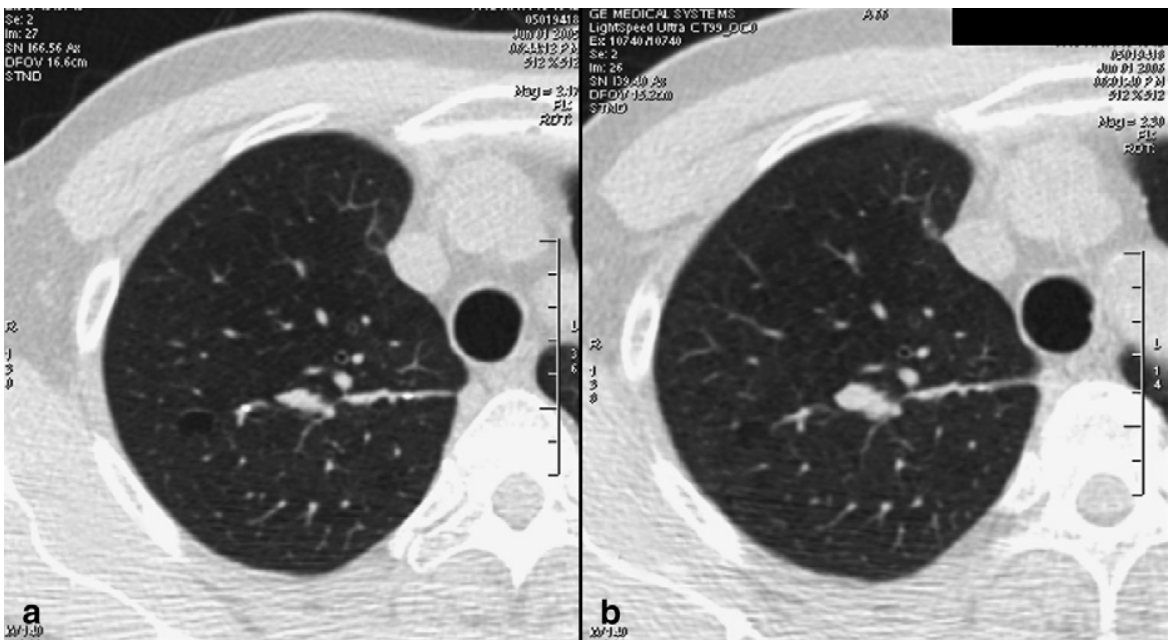

A
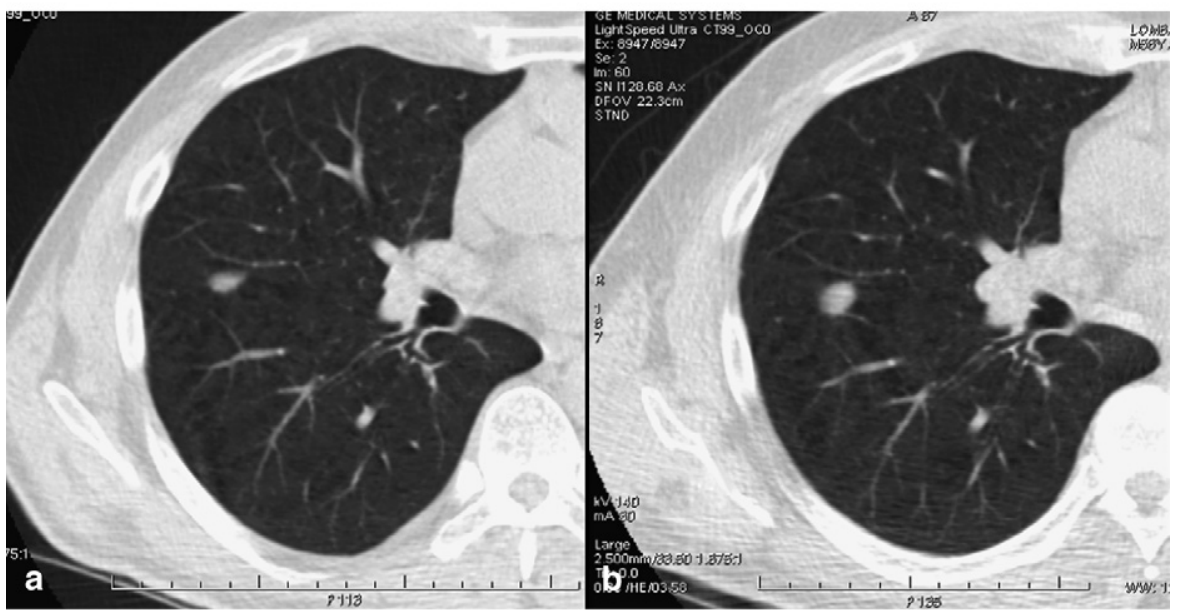

Figure 4. Examples of false-positives. In both cases, a centrally located nodule had increased in size and volume compared with baseline (a) on repeat scan (b); both were benign at surgical biopsy. A, The lesion had a doubling time of 162 days and negative PET scan. Lobectomy was necessary for removal. Histology showed chronic inflammation. B, The lesion was removed via a small lateral thoracotomy, and pathologic examination documented a scissural lymph node.
CAD is not part of the routine diagnostic approach because its use is not well established in EIO. In the future, the routine application of this software method is likely to improve accuracy for the measurement of such nodules, and it is hoped that experience and technical improvements will further increase the sensitivity and accuracy of the technique.

The present study indicates that CT-PET is significantly less sensitive $(70 \%)$ for cancers detected at annual screenings, mainly because they are smaller and slower growing than prevalent nodules. The threshold was based on maximum pixel-standardized uptake value. As previously reported, ${ }^{9}$ the threshold was 2 at the beginning of the study, but after 1 year we found that sensitivity was increased without reducing specificity by using a lower maximum pixelstandardized uptake value threshold to 1.5 in cases of nodules less than $1 \mathrm{~cm}$ or non-solid nodules.

Another important finding of the present study was that the number of false-positive cases at surgery was greater in nodules biopsied after the annual screening scan than in prevalent nodules biopsied at baseline scan, and pathologic findings showed more chronically inflamed nodules after the first annual screening scan. Most of these false-positives occurred during the first half of the first annual screening; after that, the introduction of more restrictive criteria for surgical resection (additional LD-CT after antibiotics instead of proceeding directly to surgical biopsy in new suspicious lesions) reduced the number of surgical false-positives. In regard to the sensitivity of the ongoing protocol, it is possible that the falsenegative rate will increase over time. The evolution of diagnostic protocol algorithm for the management of pulomonary nodules until the second year of screening trial is fully described in Appendix 1.

\section{Conclusions}

Our CT screening protocol for lung cancer provides good sensitivity and specificity, with only a small proportion of screened subjects undergoing invasive diagnostic procedures 
for benign disease and a small number of cancers progressing beyond stage I. Our data indicate that the introduction of CAD and volumetry, reduction of CT-PET positivity thresholds, and accumulation of experience by our multidisciplinary nodule management team will further improve the diagnostic accuracy of the protocol.

\section{References}

1. Jemal A, Thomas A, Murray T, et al. Cancer statistics, 2002. CA Cancer J Clin. 2002;52:23-47.

2. NCI: SEER Cancer Statistics Review, 1996-2002. Accessed online January $20^{\text {th }}, 2008$.

3. Henschke CI, Yankelevitz DF, et al. Survival of patients with stage 1 lung cancer detected on CT screening. N Engl J Med. 2006;355: 1763-71.

4. Swensen SJ, Jett JR, Hartman TE, et al. Lung cancer screening with CT: Mayo Clinic experience. Radiology. 2003;226:756-61.

5. White CS, Romney BM, Mason AC, Austin JH, Miller BH, Protopapas Z. Primary carcinoma of the lung overlooked at CT: analysis of findings in 14 patients. Radiology. 1996;199:109-15.

6. New York Early Lung Cancer Action Project Investigators. CT screening for lung cancer: Diagnoses Resulting from the New York Early Lung Cancer Action Project. Radiology. 2007;243: $239-49$.

7. Takashima S, Sone S, Li F, et al. Small solitary pulmonary nodules $(<$ or $=1 \mathrm{~cm}$ ) detected at population-based CT screening for lung cancer: reliable high-resolution CT features of benign lesions. AJR Am J Roentgenol. 2003; 180:955-64.

8. Mulshine JL, Sullivan DC. Clinical practice. Lung cancer screening. N Engl J Med. 2005;352:2714-20.

9. Veronesi G, Bellomi M, Veronesi U, et al. Role of positron emission tomography scanning in the management of lung nodules detected at baseline computed tomography screening. Ann Thorac Surg. 2007;84: 959-65.

\section{Appendix 1.}

Evolution of diagnostic protocol algorithm for the management of pulmonary nodules detected at baseline CT screening from the beginning of study until the second year of screening trial. Main modification included the de-
10. Veronesi G, Bellomi M, Mulshine J, et al. Lung cancer screening with low-dose computed tomography: a non-invasive diagnostic protocol for baseline lung nodules. Lung Cancer. 2008 Feb 26 [Epub ahead of print].

11. Wiemker R, Rogalla P, Blaffert T, et al. Aspects of computer-aided detection (CAD) and volumetry of pulmonary nodules using multislice $\mathrm{CT}$. Br J Radiol. 2005;78(Spec No 1):S46-56.

12. Jett JR. Limitations of screening for lung cancer with low dose spiral computer tomography. Clin Cancer Res. 2005;11:4988s-92s.

13. Bach PB, Jett JR, Pastorino U, et al. Computed tomography screening and lung cancer outcomes. JAMA. 2007;297:953-61.

14. National Lung Screening Trial website. Available at: http://www. nci.nih.gov/nlst/. Accessed online January $20^{\text {th }}, 2008$.

15. Flehinger BJ, Kimmel M, Melamed MR. The effect of surgical treatment on survival from early lung cancer. Implications for screening. Chest. 1992;101:1013-8.

16. Sugarbaker DJ, Strauss GM. Extent of surgery and survival in early lung carcinoma: implications for overdiagnosis in stage IA nonsmall cell lung carcinoma. Cancer. 2000;89(11 Suppl):2432-7.

17. Sobue T, Suzuki T, Matsuda M, Kuroishi T, Ikeda S, Naruke T, The Japanese Lung Cancer Screening Research Group. Survival for clinical stage I lung cancer not surgically treated. Comparison between screendetected and symptom-detected cases. Cancer. 1992;69:685-92.

18. Flieder DB, Vazquez M, Carter D, et al. Pathologic findings of lung tumors diagnosed on baseline CT screening. Am J Surg Pathol. 2006;30: 606-13.

19. Carter D, Vazquez M, Flieder DB, et al. ELCAP, NY-ELCAP. Comparison of pathologic findings of baseline and annual repeat cancers diagnosed on CT screening. Lung Cancer. 2007;56:193-9.

20. Pajares MJ, Zudaire I, Lozano MD, et al. Molecular profiling of computed tomography screen-detected lung nodules shows multiple malignant features. Cancer Epidemiol Biomarkers Prev. 2006;15:373-80.

21. Bianchi F, Hu J, Pelosi G, et al. Lung cancers detected by screening with spiral computed tomography have a malignant phenotype when analyzed by cDNA microarray. Clin Cancer Res. 2004;10(18 Pt 1): $6023-8$.

creased maxSUV threshold for small and non-solid nodules and the separation between solid or partially solid nodules and non-solid nodules in terms of interval of follow-up.

Time 0

Nodules $<5 \mathrm{~mm}$ : LD-CT in $1 \mathrm{y}$

Nodules 5-8 mm: LD- CT at $3 \mathrm{mo}$

Nodules $>8 \mathrm{~mm}$ : CT-PET (maxSUV threshold 2)

$12 \mathrm{mo}$

Nodules $<5 \mathrm{~mm}$ : LD-CT in $1 \mathrm{y}$

Nodules 5-8 mm: LD-CT at $3 \mathrm{mo}$

Nodules $>8 \mathrm{~mm}$ : LD-CT at $1 \mathrm{mo}$ after antibiotics, if stability or progression: CT-PET

Max SUV threshold $>2$ for nodules $>1 \mathrm{~cm}$ and solid or partially solid

Max SUV threshold $>1.5$ for nodules $<1 \mathrm{~cm}$ or non-solid lesions.

$24 \mathrm{mo}$

Solid or partially solid nodules

Nodules < $5 \mathrm{~mm}$ : LD-CT in 1 y

Nodules 5-8 mm: LD-CT at $3 \mathrm{mo}$

Nodules $>8 \mathrm{~mm}$ : LD-CT at $1 \mathrm{mo}$ after antibiotics if stability or progression: CT-PET

Non-solid nodules

Nodules $<8 \mathrm{~mm}$ follow-up 1 y

Nodules $>8 \mathrm{~mm}$ : LD-CT at 3 mo with antibiotics, if increased in diameter or density biopsy, if stable LD-CT 1 y

Max SUV threshold $>2$ for nodules $>1 \mathrm{~cm}$ and solid or partially solid

Max SUV threshold $>1.5$ for nodules $<1 \mathrm{~cm}$ or non-solid lesions

LD-CT, Low-dose computed tomography; CT-PET, computed tomography-positron emission tomography; maxSUV, maximum pixel-standardized uptake value. 